\title{
THE SYMIMETRY GROUP OF A CURVE PRESERVES A PLANE
}

\author{
ROBERT GULLIVER AND FRANK MORGAN ${ }^{1}$
}

\begin{abstract}
It is shown that the symmetries of a closed curve without self-intersections in euclidean or hyperbolic $n$-space have an invariant plane in common. This allows a complete characterization of the symmetry group.
\end{abstract}

TheOREM. Let $\Gamma$ be a Jordan curve in $\mathbf{R}^{n}$. Let $\mathbf{G}$ be its group of symmetries, that is, of isometries of $\mathbf{R}^{n}$ which leave $\Gamma$ invariant. Then there is a two-dimensional plane $\pi$ invariant under $G$. In case $\Gamma$ spans $\mathbf{R}^{n}, G$ is a finite cyclic group, is the group of symmetries of a regular polygon, or is isomorphic to the symmetry group $\mathbf{O}(2)$ of a plane cirçle.

It may be observed, with the help of simple examples, that each abstract group listed in the statement of the theorem is realized as the full symmetry group of an appropriate Jordan curve in $\mathbf{R}^{\mathbf{2}}$. Moreover, each finite group in the list occurs as the symmetry group of a curve which spans $\mathbf{R}^{n}$, for any $n>2$, while $\mathbf{O}(2)$ occurs only when $n$ is even. In the more general case of a Jordan curve whose affine span is a $k$-dimensional subspace $L$ of $\mathbf{R}^{n}$, it follows immediately from the theorem (and the fact that $G$ fixes a point in $\pi$ ) that $G=G_{1} \times O(n-k)$, where $G_{1}$ is one of the groups listed in the theorem and $\mathbf{O}(n-k)$ acts as the identity transformation on $L$. The special case of a Jordan curve in $\mathbf{R}^{3}$ was stated, without proof, by Wente in [1]. Wente examined the question whether a minimal surface of the topological type of the disk, bounded by $\Gamma$ and having smallest area, must also be invariant under the group $G$.

The theorem may be adapted without serious difficulty for the context of hyperbolic or affine geometry, as outlined at the end of this paper.

Proof. Choose an orientation for $\Gamma$ and put

$$
G_{0}=\{g \in G: g \text { preserves the orientation of } \Gamma\} .
$$

$G_{0}$ is a subgroup of $G$, and if $\sigma \in G-G_{0}$, then $G=G_{0} \cup \sigma G_{0}$. We claim that

$$
\text { if } g \in G_{0} \text { leaves a single point } p \in \Gamma \text { fixed, then } g=\mathrm{id} \text {. }
$$

Since $\Gamma$ spans $\mathbf{R}^{n}$, it suffices to show that if $q$ is any other point in $\Gamma$, then $g(q)=q$. Assume $g(q) \neq q$. Since $g$ is an orientation-preserving homeomorphism of $\Gamma$, the points $q, g(q), g^{2}(q), \ldots$ lie in order along an arc of $\Gamma$ from $q$ to $p$, and

Received by the editors August 27, 1980 and, in revised form, April 22, 1981.

1980 Mathematics Subject Classification. Primary 53A04.

${ }^{1}$ Research partially supported by National Science Foundation grants MCS 79-02032 and MCS 79-22051. 
hence the sequence must converge. But since each $g^{i}$ is an isometry,

$$
\left|g^{i}(q)-g^{i+1}(q)\right|=|q-g(q)| \nrightarrow 0,
$$

a contradiction, proving (1).

To complete the proof, we consider the cases $G$ finite and $G$ infinite.

Case 1. $G$ finite. Firstly, we claim that $G_{0}$ is cyclic. Let $p$ be any point on $\Gamma$. Choose $g_{0} \in G_{0}$ such that $g_{0}(p)$ is the first point of the orbit $G_{0} p$ after $p$ along $\Gamma$. Since $g_{0}$ is an orientation-preserving homeomorphism of $\Gamma$ and $g_{0}$ carries $G_{0} p$ to itself, the points of $G_{0} p$ along $\Gamma$ in order are precisely $g_{0}(p), g_{0}^{2}(p), \ldots, g_{0}^{k}(p)=p$. It follows from (1) that $G_{0}=\left\{g_{0}^{i}: 0 \leqslant i<k\right\}$.

Secondly, we show that if $\sigma \in G-G_{0}$, then

$$
g_{0} \sigma g_{0}=\sigma \text {. }
$$

Since $\sigma$ reverses orientation, it leaves fixed two points of $\Gamma$, and we can assume $\sigma(p)=p$. Since

$$
\sigma\left(G_{0} p\right)=\left(\sigma G_{0} \sigma\right) p=G_{0} p,
$$

and since $g_{0}(p)$ and $g_{0}^{-1}(p)$ are the points of $G_{0} p$ immediately following and preceding $p$ on $\Gamma$,

$$
\sigma\left(g_{0}(p)\right)=g_{0}^{-1}(p)=g_{0}^{-1} \sigma(p) .
$$

As an element of $G_{0}$ leaving $p$ fixed, $\sigma^{-1} g_{0} \sigma g_{0}=\mathrm{id}$, and (2) is proved. Hence, either $G=G_{0}$ (a cyclic group), or $G$ is a dihedral group (the symmetry group of a regular polygon in $\mathbf{R}^{2}$ ).

Finally, we will find a plane $\pi$ invariant under $G$. Since the problem is trivial if $G=G_{0}$ or $\left|G_{0}\right|=1$, we assume $|G|=2\left|G_{0}\right|$.

By (3) the polygon $P$ with vertices $p, g_{0}(p), \ldots, g_{0}^{k-1}(p)$ is invariant under $G$. The symmetries of $P$, including the symmetries of $\Gamma$, act as isometries on the span $S$ of $P$. It suffices to find a plane in $S$ invariant under the group of symmetries of $P$ in $S \cong \mathbf{R}^{m}$. We shall assume $m>2$, since for $m<2$ the result is obvious.

We choose the center of gravity of $P$ as the origin. Then $g_{0} \in \mathbf{O}(m)$, and for some orthonormal pair of vectors $u, v \in \mathbf{R}^{m}$, and for some $\theta \in \mathbf{R}, g_{0}(u)=\cos \theta u$ $-\sin \theta v$ and $g_{0}(v)=\sin \theta u+\cos \theta v$. Equivalently, $g_{0}(w)=\lambda w$ where $w=u+i v$ and $\lambda=e^{i \theta}$. We may assume $\theta$ is not a multiple of $2 \pi$. Let $z=w+\overline{\sigma(w)}$. Then using the relation (2), we see that $g_{0}(z)=\lambda z$. Since $\sigma^{2}=\mathrm{id}$, we have also that $\sigma(z)=\sigma(w)+\bar{w}=\bar{z}$. That is, both $g_{0}$ and $\sigma$ preserve the subspace spanned by the real and imaginary part of $z$. It may happen that the real and imaginary parts of $z$ are dependent, so that a nontrivial linear combination of $\sigma(u)$ and $\sigma(v)$ lies in the plane spanned by $u$ and $v$. In this case, both $\sigma(u)$ and $\sigma(v)$ lie in that plane, since it is invariant under $g_{0}$ and since $g_{0}(\overline{\sigma(w)})=\lambda \overline{\sigma(w)}$. Thus, in either case, we have found a plane which is invariant under both $g_{0}$ and $\sigma$, and hence under all of $G$.

Case 2. $G$ infinite. As a closed subgroup of a Lie group, $G_{0}$ is itself a Lie group [2, p. 135]. Since $G_{0}$ is compact and infinite, it has dimension at least one and hence contains a subgroup of the form $G_{1}=\{\exp (t A): t \in \mathbf{R}\}$ for some $A$ in the Lie algebra. It follows from (1) that $G_{0}=G_{1}$, that $\Gamma$ is an orbit of $G_{0}$, and hence that $\Gamma$ is smooth. Now if we take the center of gravity of $\Gamma$ as the origin in $\mathbf{R}^{n}$ and replace 
$A$ by an appropriate real multiple, then $G_{0} \subset \mathbf{O}(n)$ and, for some orthonormal pair $\{u, v\}$ of vectors in $\mathbf{R}^{n}, \exp (t A)(w)=e^{i t} w$, where $w=u+i v$. Write $g_{t}=\exp (t A)$. Then $g_{t} \sigma g_{t}=\sigma$, by an argument parallel to that used to establish the relation (2). From this follows that the real and imaginary parts either of $w$ or of $z=w+\overline{\sigma(w)}$ span a plane invariant under both $\sigma$ and $g_{t}$, for all $t$, as was shown above. This plane is invariant under $G$. As an abstract group, $G$ is the group of symmetries of the circle in $\mathbf{R}^{2}$.

REMARKS. 1. We have expressed the theorem in the euclidean context for the sake of clarity. It should be observed, however, that the theorem remains valid, with an analogous proof, when $\mathbf{R}^{n}$ is replaced by $n$-dimensional hyperbolic space $H^{n}$. In this context, the theorem asserts the existence of an invariant two-dimensional, totally geodesic surface $\pi$; the same classification of $G$ is valid, under the assumption that $\Gamma$ lies in no totally geodesic hypersurface.

One element in our proof, whose meaning in hyperbolic space might not be obvious, is the notion of center of gravity. Let $\boldsymbol{H}^{n}$ be realized as the connected component $\left\{(t, x) \in L^{n+1}: t=\sqrt{1+\|x\|^{2}}\right\}$ of a hyperboloid in Minkowski space $L^{n+1}=\mathbf{R} \times \mathbf{R}^{n}$, with the Lorentz metric $d s^{2}=-d t^{2}+\|d x\|^{2}$. Then any piecewise smooth curve in $H^{n}$ has an affine center of gravity $\left(t_{0}, x_{0}\right)$ in $L^{n+1}$, with $t_{0}>0$; $\left(t_{0}, x_{0}\right)$ is not in $H^{n}$, however. We project this point toward the origin in $L^{n+1}$ to find the hyperbolic center of gravity $\left(t_{1}, x_{1}\right)=\left(t_{0}, x_{0}\right) / \sqrt{t_{0}^{2}-\left\|x_{0}\right\|^{2}}$. Now this construction is invariant under Lorentz transformations of $L^{n+1}$. But any isometry of $H^{n}$ extends to a Lorentz transformation, which shows that the hyperbolic center of gravity is well defined.

2 . In the still more general context of an ambient riemannian manifold $M^{n}$, one does not expect to find a totally geodesic two-dimensional invariant surface, as is shown by the two examples following; nonetheless, the same classification of $G$ as an abstract group is obtained, under the assumption that $\Gamma$ lies in no totally geodesic submanifold $N \neq M$. For the first example, we begin with a curve $\Gamma \subset \mathbf{R}^{n}$ having a finite symmetry group $G$. Let the euclidean metric be perturbed in a $G$-invariant fashion; then, in general, there will be no two-dimensional totally geodesic surfaces whatsoever. The second example shows that $G$ may have only odd-dimensional invariant submanifolds. This is illustrated with the choice of $\Gamma \subset M=S^{3} \subset \mathbf{R}^{4}$ as the curve described by $\theta \mapsto\left(\alpha e^{i \theta / p}, \beta e^{i \theta / q}\right) \in \mathbf{C}^{2}=\mathbf{R}^{4}$, where $p$ and $q$ are relatively prime integers and $|\alpha|^{2}+|\beta|^{2}=1$. The symmetry group $G$ of $\Gamma$ is isomorphic to $\mathbf{O}(2)$ and has infinitesimal generator $(i \alpha / p, i \beta / q)$. Its invariant submanifolds are curves congruent to $\Gamma$ and $S^{3}$ itself. Our proof fails here because $\Gamma$ has no "center of gravity" in $S^{3}$.

3. A second and more difficult direction of generalization involves the affine symmetry group of a curve $\Gamma \subset \mathbf{R}^{n}$ :

$$
G_{A}=\left\{g=h \circ T: h \in \mathrm{GL}(n), T \in \mathbf{R}^{n}, g(\Gamma)=\Gamma\right\},
$$

where $G L(n)$ fixes the origin and $\mathbf{R}^{n}$ acts by translation. As shown above, $G_{A}$ fixes the center of gravity of $\Gamma$ (or of a finite orbit in $\Gamma$ ), so that we may assume $G_{A} \subset \mathrm{GL}(n)$. 
We need to establish the property (1) above: that if $g \in G_{A}$ preserves the orientation of $\Gamma$ and fixes one point, then $g$ is the identity transformation on $\mathbf{R}^{n}$. Now $g$ may be defective, that is, its (complex) eigenvectors may fail to form a basis of $\mathbf{C}^{n}$. We shall show this is not the case. In fact, let $E$ be the subspace of $\mathbf{C}^{n}$ spanned by eigenvectors corresponding to eigenvalues of modulus one. We claim that $E=\mathbf{C}^{\boldsymbol{n}}$. Otherwise, since $\Gamma$ spans $\mathbf{R}^{\boldsymbol{n}}$, we may choose $q \in \Gamma \backslash E$. In particular, $g(q) \neq q$, and $q_{k}=g^{k}(q)$ forms a nonconstant convergent sequence, as was shown above. If $q$ is a sum of eigenvectors, then $|\lambda|>1$ leads to a contradiction; similarly, $|\lambda|<1$ would imply that $\left\{g^{-k}(q)\right\}$ is unbounded. Write $q=\Sigma_{i} a_{i} e_{i}$, where $\left\{e_{1}, \ldots, e_{n}\right\}$ is a basis with respect to which $g$ is in Jordan canonical form, and one of the coefficients $a_{m} \neq 0$, where $e_{m}$ is not an eigenvector. Assume that $\left\{e_{1}, \ldots, e_{l}\right\}$ spans the corresponding invariant subspace of $\mathrm{C}^{n}$. Thus $g\left(e_{1}\right)=\lambda e_{1}$ and $g\left(e_{j}\right)=\lambda e_{j}+e_{j-1}, 2 \leqslant j<l$. We shall assume that $m$ is the largest index, $2<m<l$, for which $a_{m} \neq 0$. We may assume further that $|\lambda|>1$, replacing $g$ by $g^{-1}$ if necessary. Introduce the euclidean norm with respect to $\left\{e_{1}, \ldots, e_{n}\right\}$, and let Pr be the orthogonal projection onto the span of $\left\{e_{m-1}, e_{m}\right\}$. We may compute

$$
g^{k}\left(e_{j}\right)=\sum_{i=0}^{j-1}\left(\begin{array}{c}
k \\
i
\end{array}\right) \lambda^{k-i} e_{j-i}, \quad \operatorname{Pr} q_{k}=\left(\lambda^{k} a_{m-1}+k \lambda^{k-1} a_{m}\right) e_{m-1}+\lambda^{k} a_{m} e_{m},
$$

and hence

$$
\begin{aligned}
\left\|q_{k}\right\|^{2} & \geqslant\left\|\operatorname{Pr} q_{k}\right\|^{2}=|\lambda|^{2 k-2}\left\{\left|\lambda a_{m}\right|^{2}+\left|\lambda a_{m-1}+k a_{m}\right|^{2}\right\} \\
& \geqslant|\lambda|^{2 k-2}\left\{\left(|\lambda|^{2}+\frac{1}{2} k^{2}\right)\left|a_{m}\right|^{2}-|\lambda|^{2}\left|a_{m-1}\right|^{2}\right\}
\end{aligned}
$$

which is unbounded, a contradiction. (We have used Young's inequality $2 k$ $\operatorname{Re}\left(\lambda a_{m-1} \bar{a}_{m}\right) \geqslant-2\left|\lambda a_{m-1}\right|^{2}-\frac{1}{2} k^{2}\left|a_{m}\right|^{2}$.) Therefore $E=\mathbf{C}^{n}$, and $g$ is an isometry in this norm, so that one may prove (1) as above.

The rest of the proof of the theorem follows essentially as before. It might be noted that the compactness of $G_{A}$ follows from the proof. The compactness implies that $G_{A}$ is conjugate to a subgroup of $O(n)$, as is well known.

4. It should be pointed out that our proof characterizes not only the abstract group structure of $G$ but also the possible representations.

\section{REFERENCES}

1. Henry Wente, The Plateau problem for symmetric surfaces, Arch. Rational Mech. Anal. 60 (1976), 149-169.

2. Claude Chevalley, Theory of Lie groups, Vol. I, Princeton Univ. Press, Princeton, N.J., 1946.

School of Mathematics, University of Minnesota, Minneapolis, MnNesota 55455

Department of Mathematics, Massachusetts institute of Technology, Cambridge, MasSACHUSETTS 02139 